\title{
Effect of manual percussion on tracheobronchial clearance in patients with chronic airflow obstruction and excessive tracheobronchial secretion
}

\author{
CP VAN DER SCHANS, DA PIERS, DS POSTMA
}

From the Department of Physiotherapy of the Department of Rehabilitation; the Department of Nuclear Medicine, and the Department of Pulmonary Disease, Academisch Ziekenhuis, Groningen, The Netherlands

ABSTRACT The effect of manual percussion of the thorax in nine patients with stable chronic airflow obstruction and excessive tracheobronchial secretion has been studied. Tracheobronchial clearance was measured over 50 minutes on three different days. On the first day manual percussion was applied for 10 minutes. In the period when percussion was applied the mucus clearance was slightly but significantly greater than in the periods when no percussion was applied. On the second day manual percussion was applied in combination with postural drainage, coughing, and breathing exercises for 20 minutes. This resulted in a much greater clearance than on the first day. On the third day postural drainage, coughing, and breathing exercises, but no manual percussion, were carried out for 20 minutes. There was no significant difference between the clearance of days 2 and 3. From this study it is apparent that manual percussion is a relatively ineffective procedure in patients with stable chronic airflow obstruction, but may be useful when the patient is not able to cough and cannot assume the appropriate position for postural drainage.

Physiotherapy is often used in the treatment of chronic airflow obstruction (CAO), especially in patients with excessive tracheobronchial secretion, ${ }^{1}$ as stasis of secretions may lead to pulmonary infection. In these circumstances physiotherapy usually consists of a combination of postural drainage, breathing exercises, coughing, and percussion. The rationale of such treatment rests on the assumption that additional mechanical and gravitational forces will help in the clearance of airway secretions.

Clinically the benefit of these methods is difficult to judge. Many studies have shown that physiotherapy including percussion, postural drainage, breathing exercises, and coughing increases the expectoration of sputum and this may lead to improvement of lung function and arterial blood gases. ${ }^{2-9}$ Postural drainage or coughing alone, however, may also increase the transport of mucus in the lungs. ${ }^{10-16}$

Measurement of lung clearance of a radioactive

Address for reprint requests: Dr CP van der Schans, University Hospital, Department of Physiotherapy of the Department of Rehabilitation, Lenshoek Kliniek (3e verd.), Oostersingel 59, 9713 EZ Groningen, The Netherlands. aerosol would appear to be the most direct objective method for evaluating the effect of chest physiotherapy. 1718

In this report we present data on the effect of manual percussion as a single procedure, as well as in combination with postural drainage, coughing, and breathing exercises, on tracheobronchial clearance in patients with chronic airflow obstruction and excessive tracheobronchial secretions.

\section{Methods}

PATIENTS

Nine men with clinically stable chronic airflow obstruction and regular daily expectoration took part in this study after giving informed consent. Approval of the university ethics committee was obtained. All patients complained of breathlessness on exertion and none had symptoms or signs suggestive of bronchiectasis. All were smokers or ex-smokers. Some characteristics of the patients are summarised in the table. All patients used their regular treatment on the days $\stackrel{\mathbb{D}}{\mathbb{O}}$ of the measurements, no extra doses were allowed. 을 Routine physiotherapy was withheld on each study day. 
Details of patients studied

\begin{tabular}{|c|c|c|c|c|c|c|}
\hline Patient No & Age (y) & $\begin{array}{l}F E V_{1} \\
\% \text { pred }\end{array}$ & $\begin{array}{l}F E V_{1} \\
\% V C\end{array}$ & Smoking $\ddagger$ & Sputum $(m l) \S$ & Treatment \\
\hline $\begin{array}{l}1^{*} \\
2 \\
3 \\
4 \\
5 \\
6 \\
7 * \\
8 \dagger \\
9\end{array}$ & $\begin{array}{l}44 \\
46 \\
50 \\
57 \\
61 \\
61 \\
61 \\
70 \\
61\end{array}$ & $\begin{array}{l}49 \\
73 \\
51 \\
52 \\
70 \\
57 \\
47 \\
51 \\
61\end{array}$ & $\begin{array}{l}44 \\
56 \\
40 \\
48 \\
45 \\
39 \\
32 \\
34 \\
37\end{array}$ & $\begin{array}{l}+ \\
+ \\
+ \\
+ \\
+ \\
+ \\
+ \\
+\end{array}$ & $\begin{array}{c}100(8) \\
60(5) \\
140(10) \\
20(2) \\
20(1) \\
120(10) \\
150(9) \\
60(6) \\
80(7)\end{array}$ & $\begin{array}{l}\text { BIT } \\
\text { BIT } \\
\text { BIT } \\
\text { BI } \\
\text { BIT } \\
\text { BI } \\
\text { BI } \\
\text { IT } \\
\text { BIT }\end{array}$ \\
\hline
\end{tabular}

*protocol 1 not completed.

tprotocol 3 not completed.

+ indicates smoker and - indicates ex-smoker.

\$Mean (SD) sputum production/day of three consecutive days.

FEV $_{1} \%$ pred-forced expiratory volume in one second as a percentage of the predicted value; $\mathrm{FEV}_{1} \% \mathrm{VC}-\mathrm{FEV}_{1}$ as a percentage of the inspiratory vital capacity; B-beclomethasone; I-ipratropium bromide; T-theophylline.

\section{RADIOAEROSOL INHALATION AND}

REGISTRATION OF BRONCHIAL CLEARANCE

A technetium $\left({ }^{99 \mathrm{~m}} \mathrm{Tc}\right)$ aerosol was administered with an air jet nebuliser connected, in side stream operation, to an intermittent positive pressure breathing respirator (Bennet AP-5). In this way a heterodisperse aerosol was produced (95\% of particles 1-15 $\mu \mathrm{m})$. After full explanation to the patient and practice inhalations for becoming accustomed to the respirator, the nebuliser was filled with $1-1.5 \mathrm{mCi}$ $(35-50 \mathrm{MBq})^{99 \mathrm{~m}} \mathrm{Tc}$ tin colloid (Amersham). After 40-50 inhalations (inspiratory pressure $<10 \mathrm{~cm} \mathrm{H}_{2} \mathrm{O}$ ) $10-15 \%$ of the tracer was deposited on the airway mucus (fig $1 b$ and $1 c$ ). The tracer remaining in the mouth and throat was removed by rinsing and swallowing water. With this method the radiation dose to the lungs is $60 \mathrm{mrad}$ and the effective total body radiation dose amounts to $10 \mathrm{mrad}(0.1 \mathrm{mGy}) .^{19}$

Before and after the radioaerosol inhalation peak expiratory flow (PEF) was determined to assess possible effects of the procedure on bronchial narrowing and to ensure comparable baseline pulmonary function on the days of measurement.
The patients were studied in the supine position. A gamma camera with a large field of view, positioned under the patient's chest and linked to a computer, was used for acquisition of one minute frames every 10 minutes over a period of 50 minutes. Subsequently over each lung a central (hilar) and a peripheral region was determined (fig $1 a$ ) visually, by using a light pen. Care was taken to use comparable areas in the three studies on each patient. The rate of disappearance of isotope from central and peripheral regions of both lungs was recorded and, after correction for physical decay, data were expressed as percentages of the starting values.

\section{STATISTICAL TECHNIQUES}

Differences in radioaerosol clearance at each 10 minute interval were compared by means of the paired Student's $t$ test; $\mathrm{p}<0.05$ was taken as indicating a significant difference.

\section{PHYSIOTHERAPY}

Physiotherapy was always given by the same physiotherapist. Measurements of tracheobronchial
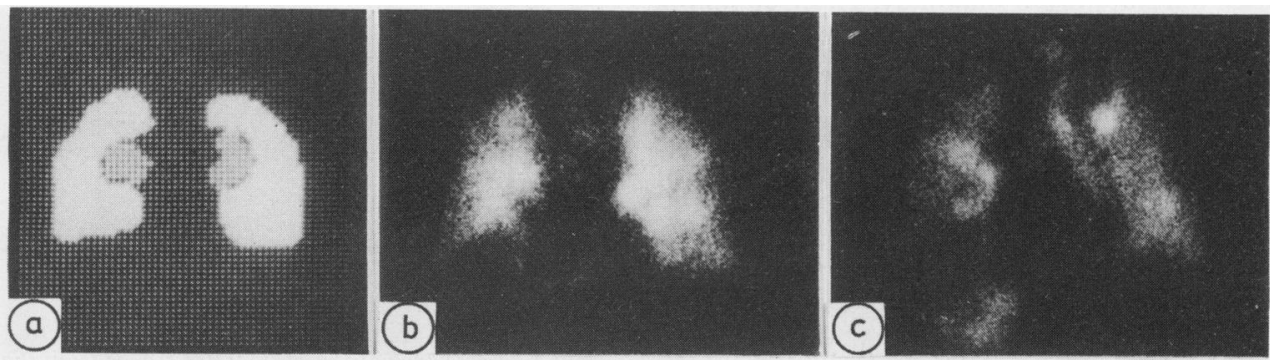

Fig 1 (a) Definition of central (darker) and peripheral (lighter) region; (b and c) example of deposition in one subject before and after physiotherapy using protocol 2 (manual percussion, breathing exercises, coughing, and postural drainage). 


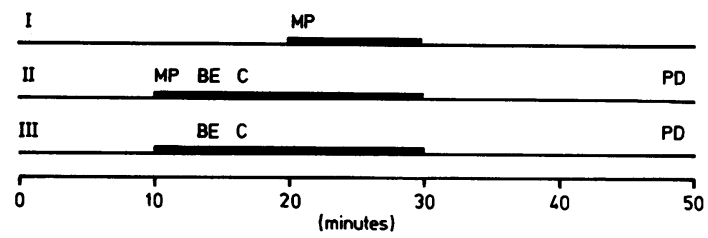

Fig 2 The three different physiotherapy protocols. $M P$ - manual percussion; $B E$ - breathing exercises; $C$-coughing; $P D$-postural drainage.

clearance were made over 50 minutes, always between $1 \mathrm{pm}$ and $3 \mathrm{pm}$. The methods of physiotherapy were manual percussion, postural drainage, breathing exercises, and coughing. The different protocols were not used in a randomised order. All patients were accustomed to different types of physiotherapy; a learning effect was therefore improbable.

The following three protocols were performed on separate days (fig 2):

1 During the first day the patient was lying horizontally in the supine position. For the first $\mathbf{2 0}$ minutes he breathed quietly, manual percussion was then applied for 10 minutes, followed by a further 20 minutes of undisturbed breathing. Manual percussion was performed with cupped hands ${ }^{1}$ on the ventral side of the thorax at a frequency of about 180 beats a minute.

2 On the second day the patient lay supine in a $20^{\circ}$ head down position. For the first 10 minutes he breathed quietly; this was followed by 20 minutes of manual percussion, breathing exercises, coughing, and rest (each for one minute and repeated five times). Thereafter the patient again breathed quietly for 20 minutes. Postural drainage was performed with the patient lying in the supine $20^{\circ}$ head down position. During the breathing exercises the patient was asked to breathe deeply in a relaxed manner. If he was wheezing he was instructed to practice lip pursing. During the coughing period the patient was asked to cough as productively as possible. During expiration the thorax was supported manually. After coughing the patient drank some water to clear the oesophagus of radioactive tracer.

3 On the third day the same protocol as on day 2 was used, but without percussion; additional one minute rest periods were substituted for the periods of manual percussion.

\section{Results}

There was no significant difference in PEF between the three days of measurement; both before and after radioaerosol inhalation mean values were 266 (SE 93) $1 \mathrm{~min}^{-1}$.

\section{MANUAL PERCUSSION VERSUS QUIET}

\section{BREATHING}

The first protocol was completed by only seven patients as two patients were unable to lie horizon- $\frac{\bar{\sigma}}{\bar{c}}$ tally for 50 minutes (table). During this day (figs $3 \vec{\nabla}$ and 4, graph 1) the clearance of the radioaerosol tracer was greater in the period when percussion was $i$ applied $(20-30 \mathrm{~min})$ than when the patient was $\overrightarrow{0}$ breathing quietly $(0-10,10-20,30-40$, and $40-50$ $\mathrm{min})$. With clearance from the peripheral zones the $\vec{\omega}$ percussion period was significantly different from the periods $10-20$ minutes $(p<0.01), 30-40$ minutes $(\mathrm{p}<0.01)$, and $40-50$ minutes $(\mathrm{p}<0.05)$. In the $\doteq$ central zones it was significantly different from the periods $0-10$ minutes $(p<0.02), 30-40$ minutes $(\mathrm{p}<0.02)$, and $40-50$ minutes $(\mathrm{p}<0.02)$.

PHYSIOTHERAPY VERSUS MANUAL PERCUSSION ALONE

All nine patients completed protocol 2 and comparison with protocol 1 was made for the seven patients who completed both. The clearance of the tracer after 50 minutes was significantly greater on day 2 (figs 3 and 4, graph 2), when manual percussion, postural drainage, breathing exercises, and coughing were performed, than on day 1 (figs 3 and 4, graph 1), when only manual percussion was used. The difference was significant both in the peripheral $(p<0.01)$ and in the central zones $(p<0.001)$.

PHYSIOTHERAPY WITH AND WITHOUT MANUAL PERCUSSION

The third protocol was completed by eight patients as one patient could not lie in a $20^{\circ}$ head down position for 50 minutes (table). Comparison between protocols 2 and 3 was made for the eight patients who completed both. There were no significant differences

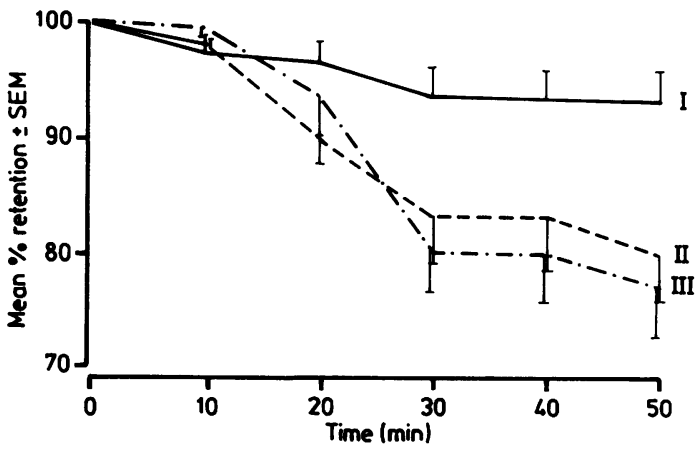

Fig 3 Clearance of the radioactive tracer from the peripheral airways expressed as percentages of the starting values (means and standard errors) on the three days of measurement $(I, I I$, and III) 


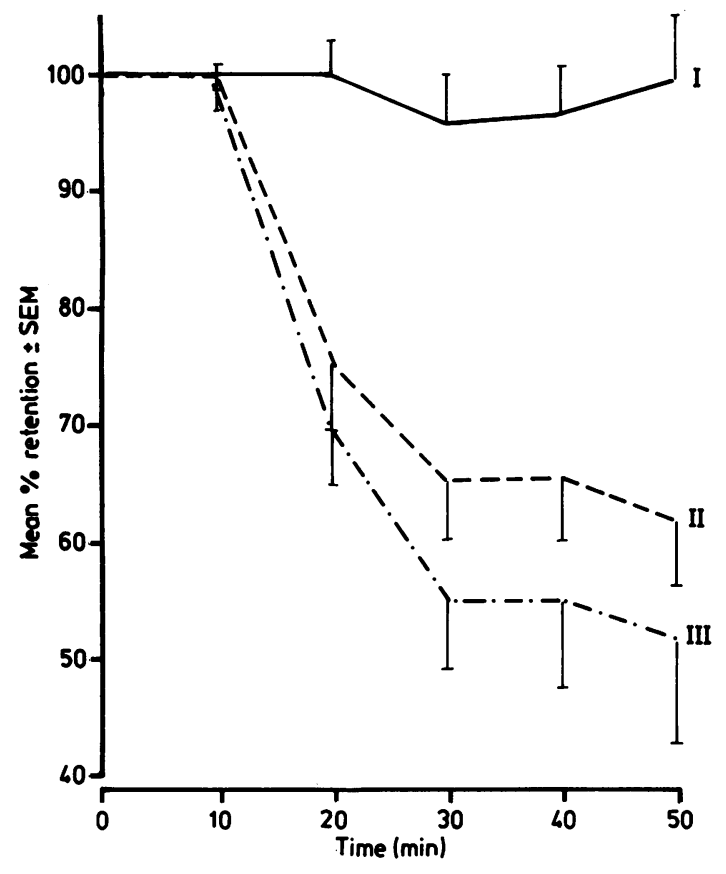

Fig 4 Clearance of the radioactive tracer from the central airways expressed as percentages of the starting values (means and standard errors) on the three days of measurement (I, II and III).

( $p>0.05$ ) between day 2 (figs 3 and 4, graph 2), when physiotherapy, including manual percussion, was applied and day 3 (figs 3 and 4, graph 3), when physiotherapy without manual percussion was applied.

\section{Discussion}

The results of this study show that manual percussion as a single procedure can slightly but significantly improve the tracheobronchial clearance in patients with chronic airflow obstruction and excessive secretions. Postural drainage and coughing with or without manual percussion can improve mucociliary clearance much more than manual percussion alone.

We used measurements of the clearance of a radioactive aerosol to evaluate the different physiotherapy protocols. The clearance of radioactive particles reflects the movement of the airway mucus on which they have been deposited. Peripheral deposition in part represents spillover from bronchiolar cilia in respiratory bronchioles and alveoli. We therefore divided the gamma camera fields into peripheral and central regions, but since the separation between the central and the peripheral fields was made in two dimensions the central field also contains some peripheral airways.

The increase in radioactive tracer seen between 30 and 50 minutes on day 1 in the central field (fig 4) probably reflects transport from the peripheral airways. The absence of any significant differences in mucociliary clearance between days 2 and 3 suggests that manual percussion does not add to the efficiency of the combination of postural drainage, coughing, and breathing exercises. This conclusion is similar to that of Rossman et $a^{14}$ in patients with cystic fibrosis. Bateman et $\mathrm{al}^{13}$ found that coughing alone did not accelerate peripheral lung clearance. In the present study we showed that coughing, when combined with postural drainage and breathing exercises, improves central as well as peripheral lung clearance. Because of differences in definition of the lung fields in our study and that of Bateman et al, however, direct comparison of the results may not be valid.

In conclusion, the effect of pulmonary physiotherapy in patients with chronic airflow obstruction and excessive tracheobronchial secretions probably results from a combination of coughing, postural drainage, and breathing exercises. Manual percussion may be useful when the patient is not able to cough and cannot tolerate postural drainage. Manual percussion should not, however, be the treatment of choice of physiotherapists for patients with chronic airflow obstruction in a stable phase of their disease.

We thank JD Bleeker for his stimulating support during this study and HJ Sluiter for his critical review of the manuscript. We were supported by a research grant from the Dutch Asthma Foundation.

\section{References}

1 Mackenzie CF, Ciesla N, Imle PC, Klemic N. Chest physiotherapy in the intensive care unit. Baltimore: Williams and Wilkins, 1981.

2 Anthonisen P, Riis P, Søgaard Andersen T. The value of lung physiotherapy in the treatment of acute exacerbations in chronic bronchitis. Acta Med Scand 1964;175:715-9.

3 Campbell AH, O'Connell JM, Wilson F. The effect of chest physiotherapy upon the F.E.V.. in chronic bronchitis. Med J Aust 1975; i:33-5.

4 Cochrane GM, Webber BA, Clarke SW. Effects of sputum on pulmonary function. $\mathrm{Br}$ Med J 1977;ii:1181-3.

5 Graham WGB, Bradley DA. Efficacy of chest physiotherapy and intermittent positive pressure breathing in the resolution of pneumonia. N Engl J Med 1978; 299:624-7.

6 Newton DAG, Stephenson A. Effect of physiotherapy on pulmonary function. Lancet 1978;ii:228-30.

7 Newton DAG, Bevans HG. Physiotherapy and intermittent positive pressure ventilation of chronic 
bronchitis. $\mathrm{Br}$ Med J 1978;ii:1525-8.

8 Bateman JRM, Newman SP, Daunt KM, Pavia D, Clarke SW. Regional lung clearance of excessive bronchial secretions during chest physiotherapy in patients with stable chronic airway obstruction. Lancet 1979;i:294-7.

9 May DB, Munt PW. Physiologic effects of chest percussion and postural drainage in patients with stable chronic bronchitis. Chest 1979;75:29-32.

10 Wong JW, Keens TG, Wannamaker EM, Grazier DN, Levison H, Aspin N. Effects of gravity on tracheal mucus transport rates in normal subjects and in patients with cystic fibrosis. Pediatrics 1977;60:146-52.

11 Oldenburg FA jun, Dolovich MB, Montgomery JM, Newhouse MT. Effects of postural drainage, exercise and cough on mucus clearance in chronic bronchitis. Am Rev Respir Dis 1979;120:739-45.

12 Feldman J, Traver GA, Taussig LM. Maximal expiratory flows after postural drainage. Am Rev Respir Dis 1979;119:239-45.

13 Bateman JRM, Newman SP, Daunt KM, Sheahan NF,
Pavia D, Clarke SW. Is cough as effective as chest $\vec{F}$ physiotherapy in the removal of excessive tracheo- $\stackrel{0}{\sim}$ bronchial secretions? Thorax 1981;36:683-7.

14 Rossman CM, Waldes R, Sampson D, Newhouse MT. Effect of chest physiotherapy on the removal of mucus $\overline{\bar{s}}$ in patients with cystic fibrosis. Am Rev Respir Dis $\overrightarrow{\mathbb{D}}$ 1982;126:131-5.

15 De Boeck C, Zinman R. Cough versus chest physio- ڤै therapy. Am Rev Respir Dis 1984;129:182-4.

16 Scherer PW. Mucus transport by cough. Chest 1981;8,suppl:830-3.

17 Pavia D, Sutton PP, Agnew JE, Lopez-Vidriero MT, Newman SP, Clarke SW. Measurement of bronchial mucociliary clearance. Eur J Respir Dis 1983;64,suppl 127:41-56.

18 Agnew JE, Bateman JRM, Pavia D, Clarke SW. Investigative nuclear medicine. A model for assessing bronchial mucus transport. J Nucl Med 1984;24:170-6.

19 Prato FS, Vinitski S. Radiation dose calculations for 응 inhalation of Tc-99m sulfur colloid radioaerosol. $\mathrm{J} \mathrm{Nucl}_{-}$ Med 1983;24:816-21. 